\title{
Fabrication of back contacts using laser writer and photolithography for inscribing textured solar cells
}

\author{
MURUGAIYA SRIDAR ILANGO ${ }^{1,2}$, VIJAY MONTERIO ${ }^{3}$ and SHEELA K RAMASESHA ${ }^{1, *}$ \\ ${ }^{1}$ Divecha Centre for Climate Change, Indian Institute of Science, Bangalore 560012, India \\ ${ }^{2}$ Department of Physics, Jain University, Bangalore 560069, India \\ ${ }^{3}$ Centre for Nanoscience and Engineering, Indian Institute of Science, Bangalore 560012, India
}

MS received 26 August 2014

\begin{abstract}
Semiconductor fabrication process begins with photolithography. Preparing a photo mask is the key process step in photolithography. The photo mask was fabricated by inscribing patterns directly onto a soda lime glass with the help of a laser beam, as it is easily controllable. Laser writer LW405-A was used for preparing the mask in this study. Exposure wavelength of $405 \mathrm{~nm}$ was used, with which $1.2 \mu \mathrm{m}$ feature size can be written in direct write-mode over the soda lime glass plate. The advantage of using the fabricated mask is that it can be used to design back contacts for thin film Photovoltaic (PV) solar cells. To investigate the process capability of LW405-A, same pattern with different line widths was written on soda lime glass samples at different writing speeds. The pattern was inscribed without proximity effect and stitching errors, which was characterized using optical microscope and field emission scanning electron microscope (FE-SEM). It was proven that writing speed of a mask-writer is decided according to the intended feature size and line width. As the writing speed increases, the edges of the patterns become rougher due to uneven scattering of the laser beam. From the fabricated mask, the solar cell can be developed embedding both the contacts at the bottom layer, to increase the absorption of solar radiation on the top surface effectively by increasing light absorption area.
\end{abstract}

Keywords. Solar cell; mask fabrication; laser writer; LW405-A; writing speed; back contact.

\section{Introduction}

The state-of-the-art photovoltaic (PV) cell has many layers of different materials stacked one above the other, with the electrode at the top of the cell to carry the charge carriers that are liberated by PV cell upon exposure to solar radiation. Because of these multiple layers, the active semiconductor junction receives only a fraction of the total solar energy that the surface of the cell receives. The major culprits are the transparent conducting oxide (TCO) layer and the metal contacts that run over the TCO layer. An attempt is being made in this study to eliminate the top contacts completely and introduce both the contacts, one for the n-type conductor and the other for the p-type conductor, at the bottom of the cell so that more of the solar radiation can reach the semiconductors. At the same time, if the junction surface area is also increased through nanostructuring of the layers then the conversion efficiency could increase.

Lithography is a vital technique used in most of the semiconductor industries, to fabricate electronic devices. Photolithography is all about drawing desired structures on the substrate using UV light source. In photolithography, it is imperative to control the proximity effect that causes unexpected distortion on the pattern due to scattering of laser beam. Photolithography uses optical proximity correction

\footnotetext{
*Author for correspondence (sheela@ caos.iisc.ernet.in)
}

in order to recompense the error caused by diffraction on the pattern. ${ }^{1}$ One of the laser writers, Sigma7300, uses subresolution assist feature to overcome proximity correction. ${ }^{2,3}$ There are few subdivisions in direct writing technology. Some of them are droplet, energy beam, flow and tip-based direct writing. ${ }^{4}$ Direct write method is employed in designing glasses for photonic devices. ${ }^{5}$ For X-ray lithography, the pattern on the mask should be very precise, as the pattern drawn over the wafer will be the exact replica of the mask through which it is exposed. There is research going on in mask-less lithography for reducing the cost as well as time required to draw and expose the pattern. ${ }^{6}$ In few cases laser beam is also utilized for deposition, in which laser beam travels over the surface of the substrate. ${ }^{7}$ Precise positioning of laser beam is required to relocate the pattern onto the photosensitized substrate. ${ }^{8}$

In early 1980 s, research on lasers was mostly concentrated on the behaviour of the laser and how it can be used for annealing, etching, polymerization and lithography. Recently, laser was allowed to react with new materials by building devices such as laser writer for mask fabrication and also for direct writing of lithographic patterns on the sample. ${ }^{9}$ ALTA $^{\mathrm{TM}} 3000$ mask laser writer reduces the mask fabrication time by reducing the turnaround time of the laser beam. ${ }^{10}$

Laser writing has been in use since three decades. Lithography has been scaled down till $100 \mathrm{~nm}$ line width using e-beam lithography. Because of its complexity and low 
precision control, it is not used for patterns with feature size more than $3 \mu \mathrm{m}$. In the laser writer LW405-A, there are four magnification lenses ranging from $\times 5$ till $\times 40$ with the strip width of $800-100 \mu \mathrm{m}$. The minimum achievable resolution with $\times 5$ lens is $8 \mu \mathrm{m}$ and with $\times 40$ lens it is $1 \mu \mathrm{m} .^{11}$

Photoresist (PR) is a light-sensitive material, which reacts with UV light. This reaction changes the chemical structure of PR and makes it soluble or insoluble depending upon the nature of the resist. The PR on which the laser writer inscribes the pattern is AZ1518. AZ1518 is a positive PR, which becomes soluble after exposure to the laser beam. This PR reacts with light in the range $320-440 \mathrm{~nm}$ wavelength. ${ }^{12}$ After exposing the pattern on the substrate, the crucial step is development of the pattern. The developer used is AZ351B in a ratio of 1:4 (AZ351B: distilled water) for high speed development. ${ }^{13}$ For deep UV tools, fused silica is required as other type of glass substrates absorb wavelengths below $350 \mathrm{~nm}$.

Schematic representation of the internal component of LW405-A is shown in figure 1. With the help of focusing lenses, the pattern resolution varies from minimum feature size to the maximum. Motion control monitors the movement of the mask in all directions. LW405 employs three scanning modes, i.e., beam, stage and vector scan. Laser writer can move the beam of laser at different speeds.

The aim of this study is to find the exact writing speed for a definite design and to develop the novel type of back contacts for thin film PV solar cells. The concept of having back contacts for both n- and p-type semiconductors in inorganic solar cells is also described in this paper.

\section{Experimental}

The selection of the substrate depends upon factors such as absorption and expansion coefficient of the material. The linear thermal coefficient of fused silica and soda lime glass are 0.4 and $9 \mu \mathrm{m}{ }^{\circ} \mathrm{C}^{-1}$, respectively. ${ }^{14}$ Although soda lime glass expands for slight variations in ambient temperatures, it can be used as a substrate material because of the temperature controllers in the clean room facilities. The selection of the substrate also depends upon its optical absorption characteristics in the UV range. Lower the wavelength of UV light, lower is the feature size of the design that can be achieved. Any layout editor can be used for drawing the designs. A few examples are L-edit, AutoCAD and Clewin. The design to be written on the substrate was drawn using the software 'Clewin' version 2.90 in this study. Figure 2 shows the design of the mask, which was to be drawn using laser writer over the chrome-coated soda lime glass. For writing layer 1 and layer 2, two soda lime glasses are used. Layer 1 is a set of bands with alternate bands connected at opposite ends like in the planar interdigitated electrode pair, ${ }^{15}$ one for n-type and other for $\mathrm{p}$-type semiconductor to make a $\mathrm{p}-\mathrm{n}$ junction. Thus, both the electrodes are placed at the bottom of the solar cell. Layer 2 is drawn to cover one set of electrodes while depositing n-type material.

Figure 3 shows the process steps involved in writing a mask. Soda lime glass of 3-4 mm thick is pre-coated with $110 \mathrm{~nm}$ of chrome and $530 \mathrm{~nm}$ of AZ1518 PR. The glass with coatings is placed on the laser writer. The D-step of 6 in the laser writer controls the speed of writing the pattern. The mask writer can take CIF or GDS-2 format of the design. The mask writer converts the CIF or GDS-2 format into a proprietary format called LDF (laser draw format) before beginning the patterning. After writing the pattern with laser writer, photo mask is taken out of the machine and submerged into a developer solution of AZ351B (sodium hydroxide solution in DI water with ratio of 1:4) for a minute. After developing, the mask is inspected using optical microscope. The mask is then post-baked for 2

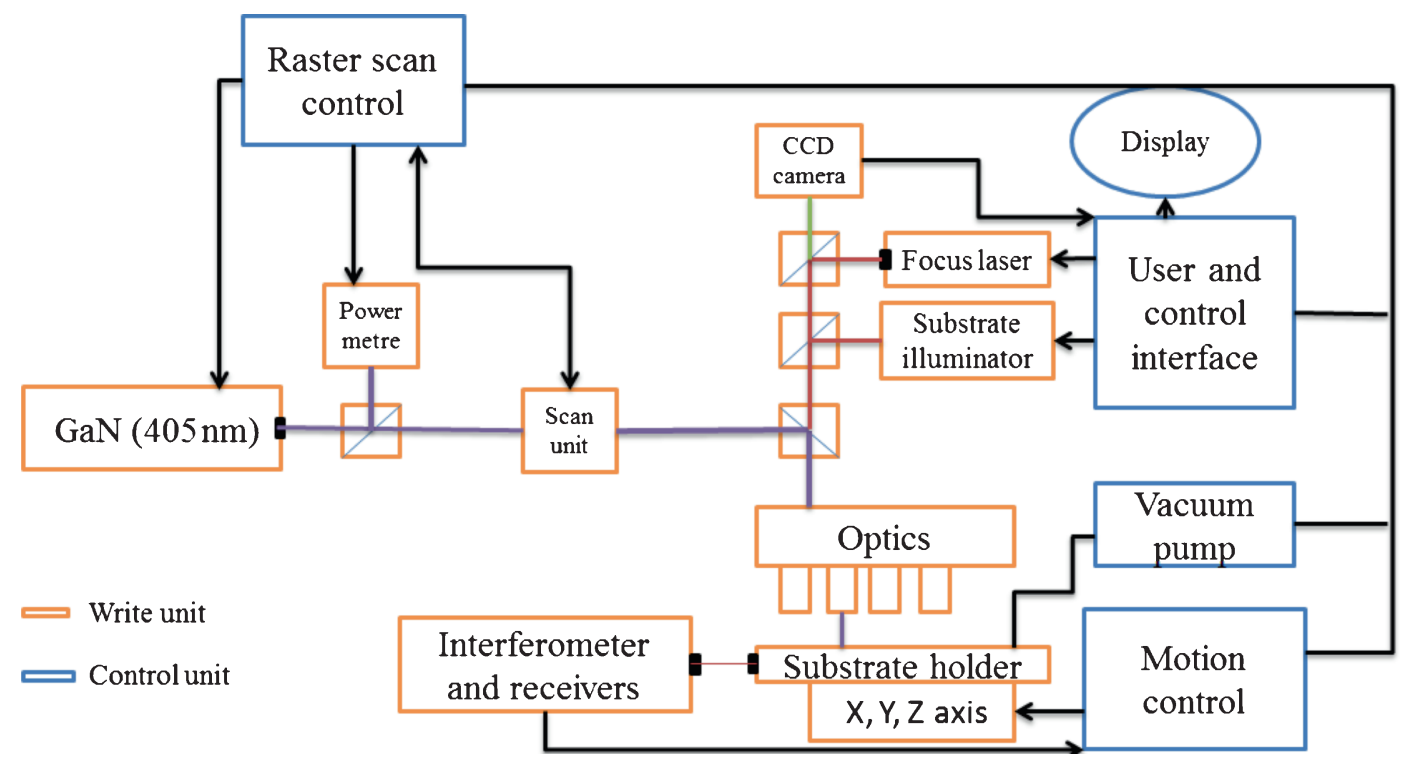

Figure 1. Blocks of LW405-A laser writer. 


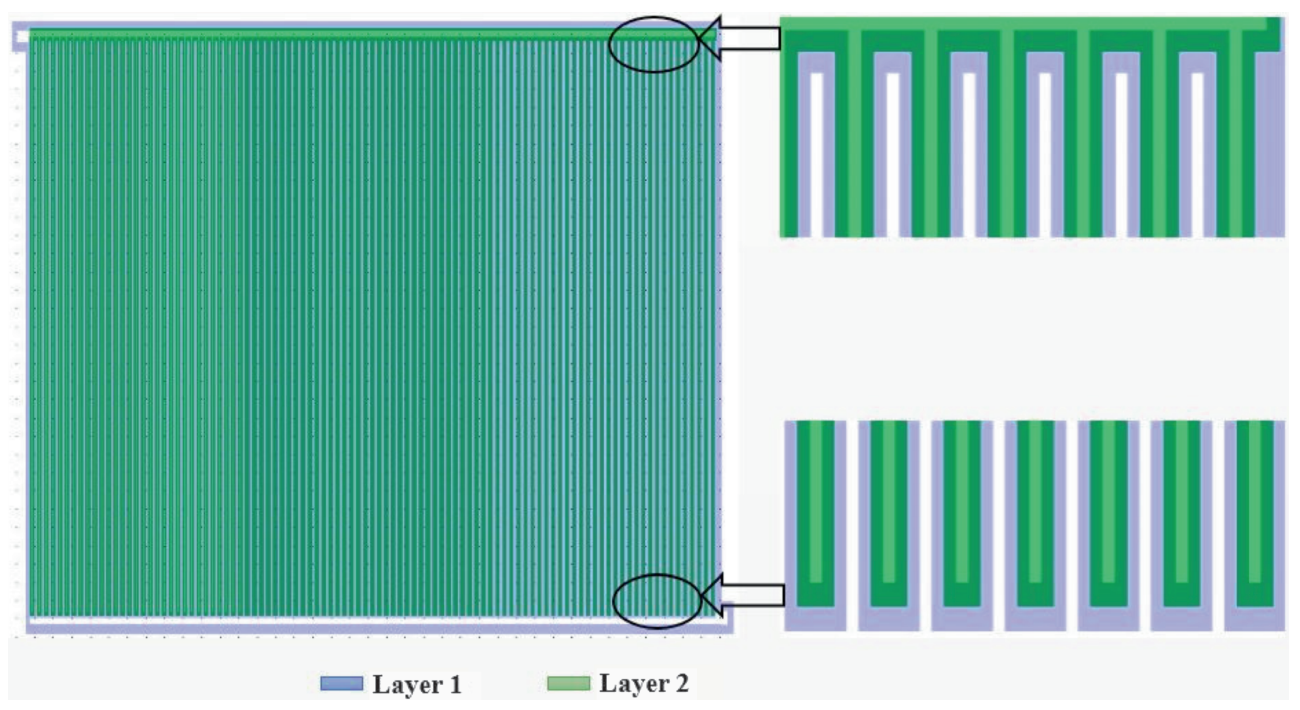

Figure 2. The pattern drawn in CleWin layout.

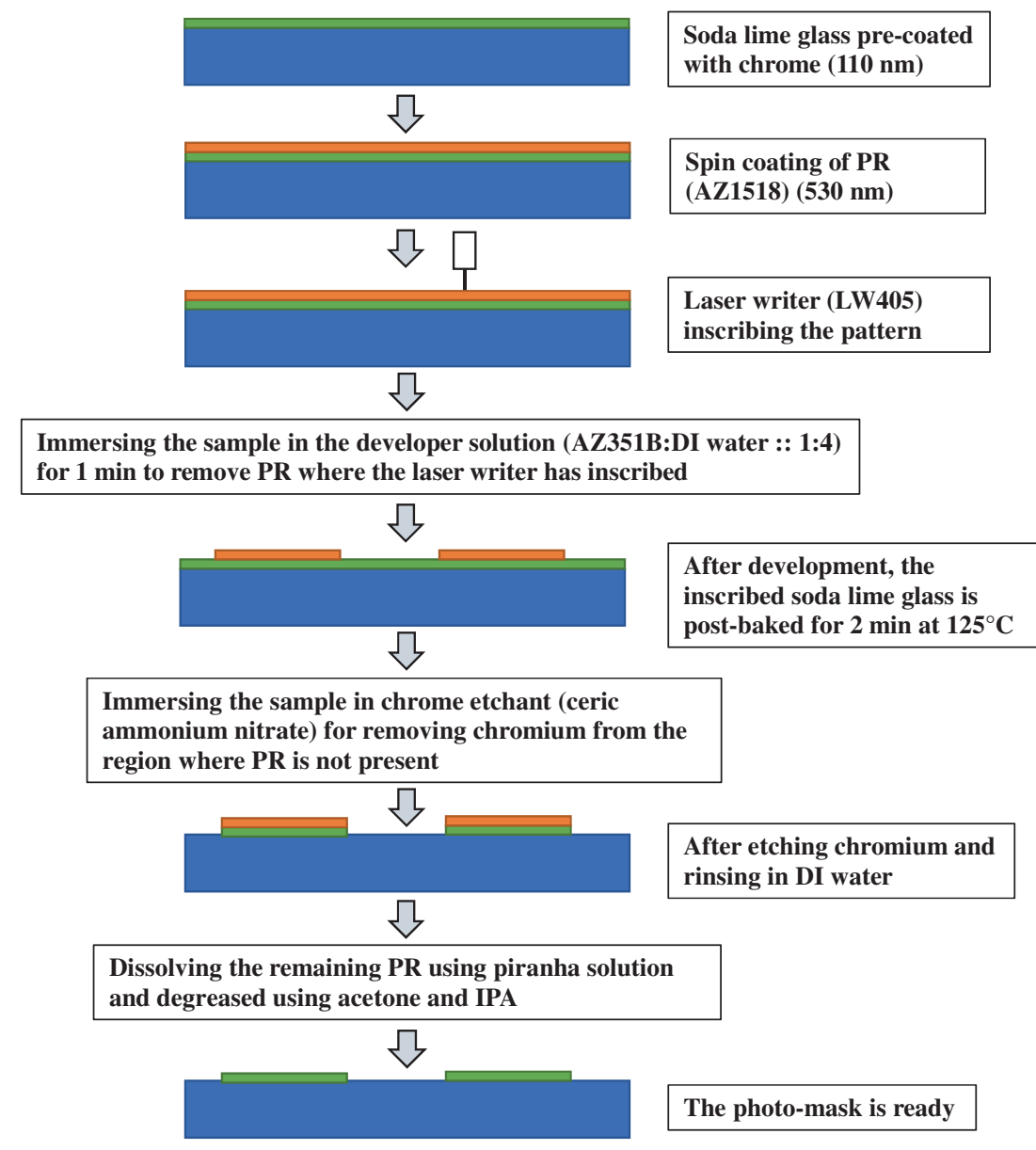

Figure 3. Schematic representation of mask writing process.

min on a hot plate at $125^{\circ} \mathrm{C}$ to harden the $\mathrm{PR}$ for next chrome etch process and introduced into chrome etchant for removing chromium from the region where resist is washed away in developer. Mask is again inspected on optical microscope for checking the features after the chrome etch.
After inspection of the mask, it is submerged in the piranha solution for $30 \mathrm{~s}$ and immediately dipped in the DI water to remove the PR. As the piranha solution, containing a combination of hydrogen peroxide and sulphuric acid $(1: 1)$, is a strong oxidizing agent, it removes the entire organic 
residue making the surface hydrophilic. Soda lime glass is then placed in acetone and isopropyl alcohol (IPA) bath for 5 min each, for degreasing it. Thus, the mask on soda lime glass is ready for use, which we designate as layer 1. For fabricating layer 2 mask, similar procedure is used except that the alternate chrome bars on the layer 1 are masked with PR.

\subsection{Solar cell fabrication}

n-Type silicon wafer with layers of silicon dioxide, chrome and gold on the top is used as a substrate for solar cell fabrication. The substrate is placed on the hot plate for dehydration bake at $250^{\circ} \mathrm{C}$ for $15 \mathrm{~min}$ and cooled. The PR S1813, which is UV sensitive and can be coated very thin on the substrate, is spin coated at a speed of 500 r.p.m. for $5 \mathrm{~s}$ and it is ramped up to 4000 r.p.m. for another 40 s. Uniform thickness of $1.1 \mu \mathrm{m}$ is obtained through spin-coating process, which is the expected thickness for $\mathrm{S} 1813$ resist. Then the substrate is soft baked at $95^{\circ} \mathrm{C}$ by placing it on a hot plate for $1 \mathrm{~min}$.

The fabricated mask on soda lime glass (layer 1) is used in the mask aligner to expose the pattern on to the silicon substrate with PR on it. In a proximity contact mode, the substrate is placed inside the mask aligner to expose it under UV light for $30 \mathrm{~s}$ with an exposure dose of $45 \mathrm{~mJ} \mathrm{~cm}^{-2}$. Then the substrate is immediately immersed in the developer solution containing DI water and AZ351B in a ratio of 4:1. The development time is 14-18 s. Later, the substrate is immersed in DI water to stop the development. Then by using gold etchant $4 \mathrm{~g}: 1 \mathrm{~g}: 40 \mathrm{ml}$ of $\mathrm{K}_{2} \mathrm{I}_{2}: \mathrm{I}_{2}: \mathrm{H}_{2} \mathrm{O}$, the gold is etched in areas where PR is not present. By following the similar procedure, layer 2 is exposed and developed before depositing n-type material.

\section{Results and discussion}

The depth of focus is affected by factors such as thickness of PR, exposure rates and energy levels of laser source. In order to reduce the effect caused by the thickness of resist, thin resist layer $(530 \mathrm{~nm})$ of AZ1518 was spin coated. If the PR layer is thicker than $530 \mathrm{~nm}$, the desired minimum line width cannot be achieved. The optical microscope images of the pattern on the soda lime glass were exact replica of the pattern drawn using CleWin layout. Different patterns with different feature sizes were drawn on soda lime glasses at two different writing speeds of 480 and $240 \mu \mathrm{m} \mathrm{s}^{-1}$. The optical micrographs of the patterns are shown in figures 4 and 5 . The pattern inscribed at $480 \mu \mathrm{m} \mathrm{s}^{-1}$ writing speed and higher exposure level of the laser beam resulted in lower control over the proximity effect (figures $4 \mathrm{a}$ and 5a). While the patterns written at $240 \mu \mathrm{m} \mathrm{s}^{-1}$ had better depth of focus (figures $4 \mathrm{~b}$ and $5 \mathrm{~b}$ ).

It is evident from the optical microscope images that patterns have rough edges when the pattern is written at higher speeds, such as $480 \mu \mathrm{m} \mathrm{s}^{-1}$. The pattern inscribed at $480 \mu \mathrm{m} \mathrm{s}^{-1}$ needed $6 \mathrm{~h}$ of writing time, while the pattern written at $240 \mu \mathrm{m} \mathrm{s}^{-1}$ required $12 \mathrm{~h}$. Also, writing feature size less than $5 \mu \mathrm{m}$ at higher speeds leads to irregularity in dimensions of the features. Proximity effects are caused due to the uneven exposure and scattering of laser beam over the edges of the pattern. If the laser beam is exposed on the substrate for a longer time, then during development the area is overdeveloped forming isotropic (blunt) edges. So considering the above facts, the writing speed of laser is selected according to the minimum feature size present in the pattern. As the pattern written at $480 \mu \mathrm{m} \mathrm{s}^{-1}$ speed is having rough edges, speed of $240 \mu \mathrm{m} \mathrm{s}^{-1}$ is used to get sharp edges.

The type of defects caused during mask fabrication are pinhole, break, chrome spot, bridging and extensions. ${ }^{16}$ By adjusting the dosage of laser beam for each pattern at different points on the mask, proximity effect can be reduced. ${ }^{17}$ If the pattern has features very close to each other, less than $1 \mu \mathrm{m}$, then there is a possibility of features merging with each other. The feature size and structure also varies during the development time. When the mask is immersed in the developer solution for less than $50 \mathrm{~s}$ the features are underdeveloped, which in turn leads to bridging error in between features. Hence, the development time is crucial in the process of fabricating micro devices.
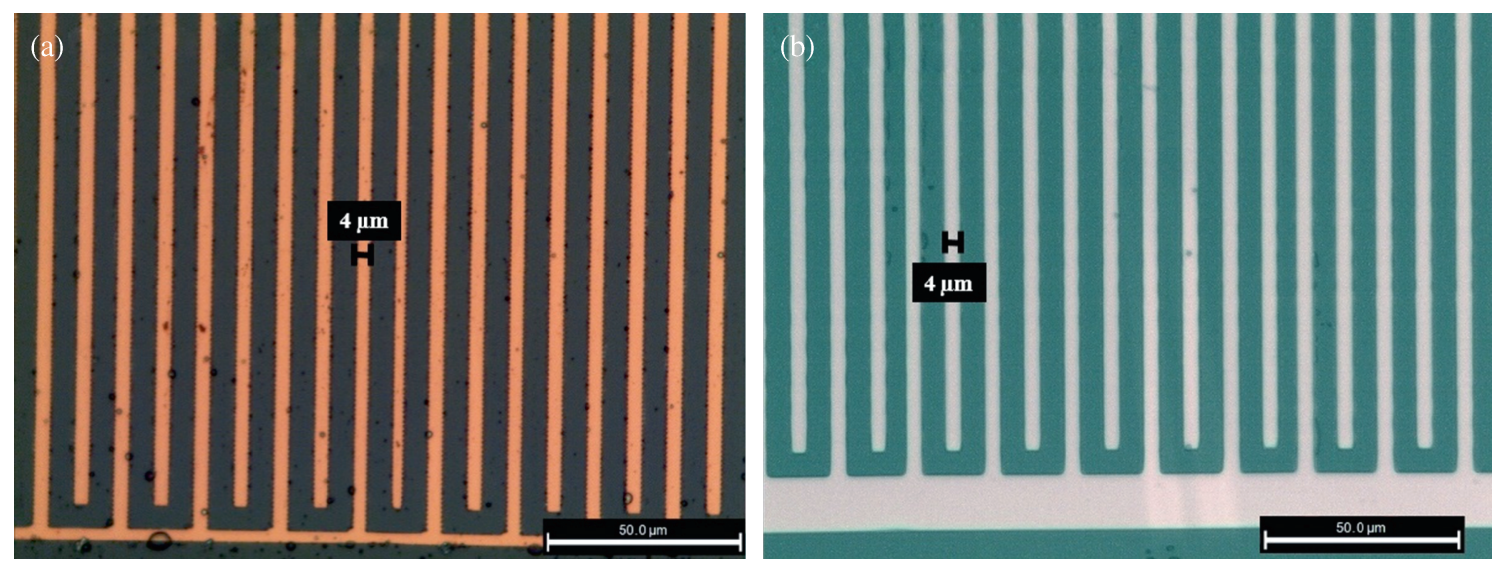

Figure 4. Optical microscopic image of denser patterns on the mask written at (a) 480 and (b) $240 \mu \mathrm{m} \mathrm{s}^{-1}$ (layer 1). 

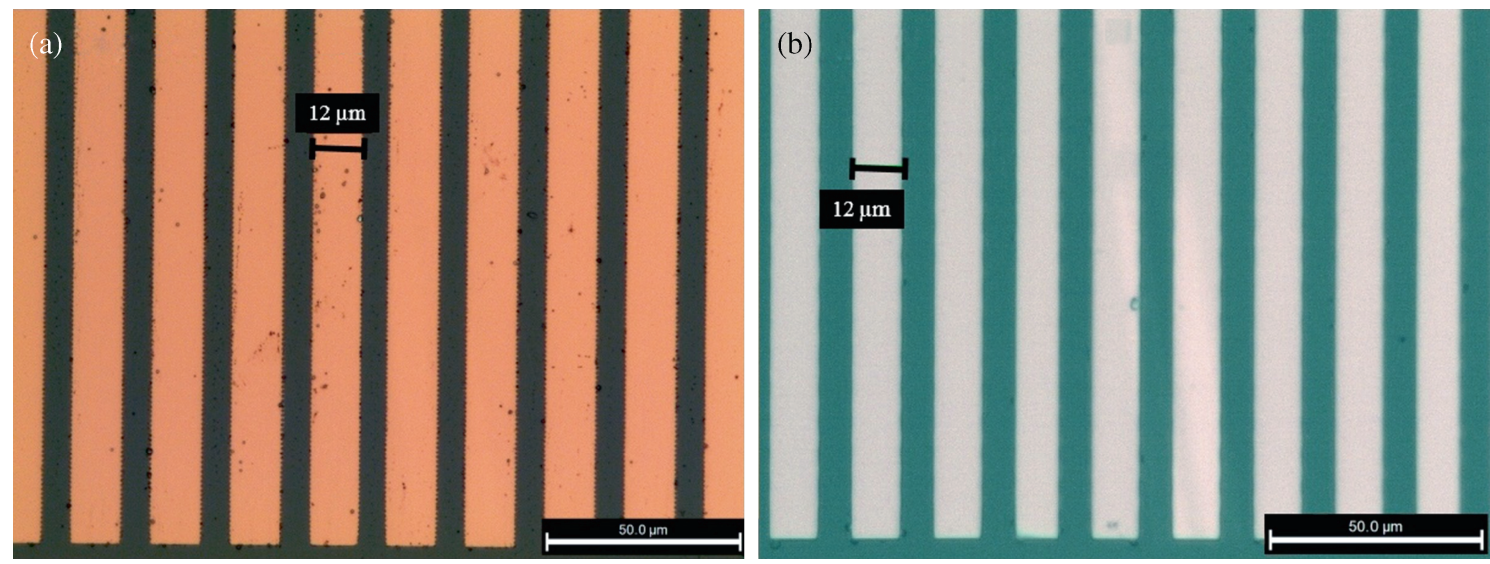

Figure 5. Optical microscopic image of denser pattern on the mask written at (a) 480 and (b) $240 \mu \mathrm{m} \mathrm{s}^{-1}$ (layer 2).

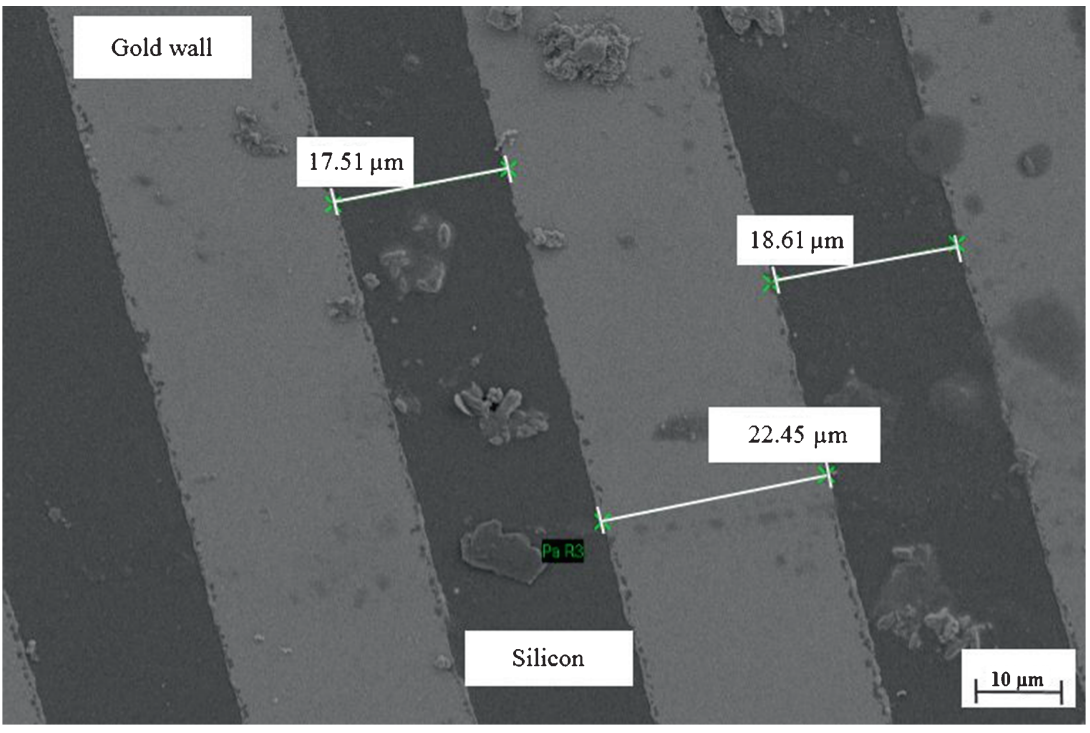

Figure 6. FE-SEM image of alternative gold walls.

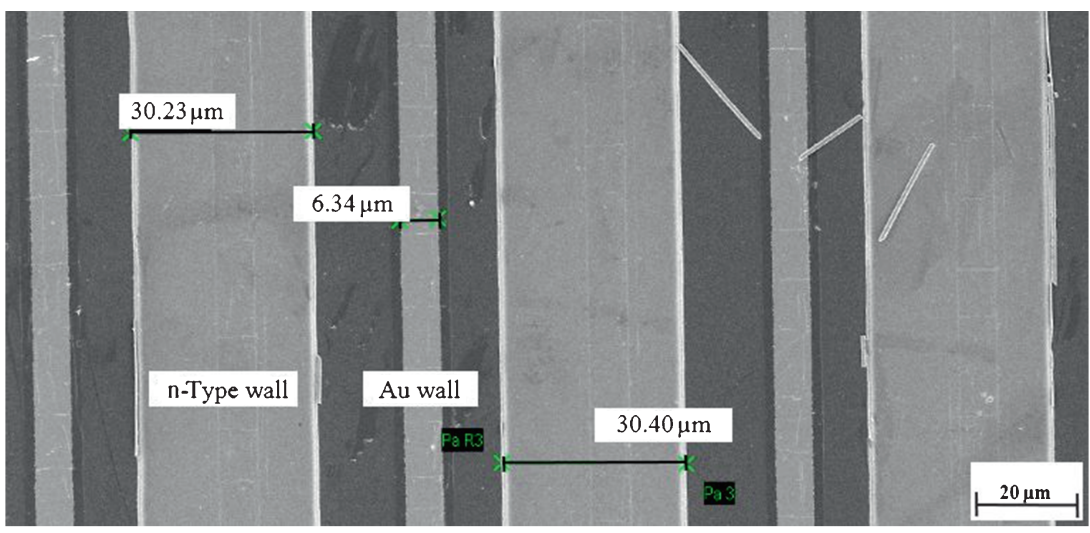

Figure 7. FE-SEM image of n-type semiconductor deposited on alternative gold walls. 


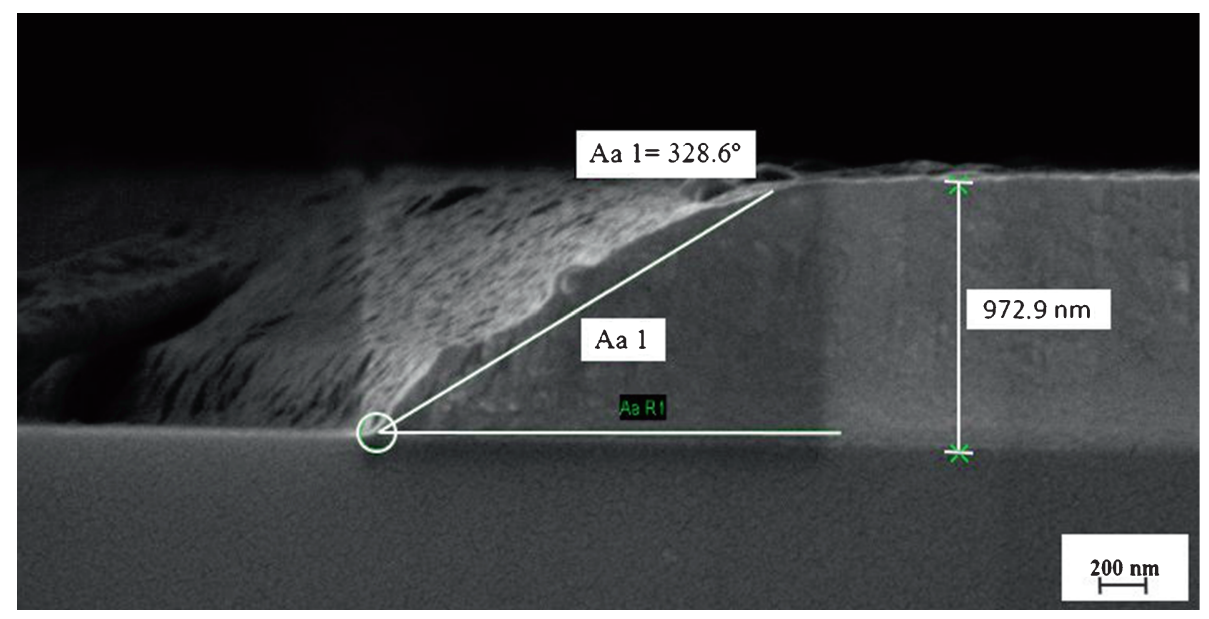

Figure 8. Cross-sectional view showing isotropic edge after sputter deposition and lift-off.

Layer 1 photo mask is exposed on to the substrate using mask aligner for the purpose of exposing the undeveloped areas to etch gold from the substrate (figure 6). Then layer 2 photo mask is exposed to uncover the areas where n-type semiconductor is to be deposited (figure 7) using sputtering process. The fabricated sample measures $6 \mu \mathrm{m}$ width of gold lines and 31 microns width of n-type semiconductor walls. The cross-sectional view shows the height of the n-type material deposited to be $972 \mathrm{~nm}$ with an isotropic edge (figure 8).

Later the p-type material can be deposited over the entire cell. Gold lines in between without n-type material on them will serve as the electrode for p-type semiconductor. Thus, the alternating gold walls are in contact with the p-type semiconductor. By annealing the sample at appropriate temperatures $\mathrm{p}-\mathrm{n}$ junction can be formed. The idea of having the contacts at the floor of the solar cell is to avoid the blockage of solar radiation by the contact material, which is usually present on the top, for conventional PV cells.

\section{Conclusion}

LW405 laser writer was examined with two different writing speeds in order to make masks for fabricating nanostructured solar cells with contacts for both $\mathrm{n}$ - and $\mathrm{p}$-type semiconductors at the base of the device. In conclusion, it was found that writing speed that needs to be used depends upon the feature size and line width. By increasing the writing speed from 240 to $480 \mu \mathrm{m} \mathrm{s}^{-1}$, the pattern obtained consist of anisotropic edges. Higher the writing speed, rougher are the edges. However, for patterns with minimum line width of less than $3 \mu \mathrm{m}$ can be written at higher writing speed to avoid bridging errors in the mask, but rough edges would still exist. LW405 is built with an interferometer, which helps in precise movement of the stage. The mask obtained is capable of fabricating $0.6 \times 0.5 \mathrm{~cm}^{2}$ novel thin film PV cells.

\section{References}

1. Noppachai A and Lee S-Y 2004 Proceedings of the 18th International Parallel and Distributed Processing Symposium (IPDPS'04)

2. Lee H-J, Nam D-S, Lee S-H, Kim H-S, Lee J-H, Han H-S, Kim B-G, Choi S-W and Han W-S 2006 Proceedings of SPIE $\mathbf{6 2 8 3}$ $62831 \mathrm{~J}-1$

3. Paufler J, Brunn S T, Körner T and Kühling F 2001 Microelectron. Eng. 57-58 31

4. Hon K K B, Li L and Hutchings I M 2008 CIRP Annals Manufacturing Technology 57601

5. Shishkin I I, Samusev K B, Rybin M V, Limonov M F, Kivshar Y S, Gaidukeviciute A, Kiyan R V and Chichkov B N 2012 Phys. Solid State $\mathbf{5 4} 1975$

6. Pease R F 2005 Microelectron. Eng. 78-79 381

7. Lehmann O and Stuke M 1994 Mater. Lett. 21131

8. Ulrich $\mathrm{H}$, Wijnaendts-van-resandt $\mathrm{R} \mathrm{W}$, Rensch $\mathrm{C}$ and Ehrensperger W 1987 Microelectron. Eng. 677

9. Chrisey D B, Pique A, Fitz-Gerald J, Auyeung R C Y, Mcgill R A, Wu H D and Duignan M 2000 Appl. Surface Sci. 154-155 593

10. Grenon B J, Defibaugh D C, Sprout D M and Taft C J 1995 Proceedings of SPIE, 15th Annual BACUS Symposium on Photomask Technology and Management 262114

11. Sundararajan A D and Rezaul Hasan S M 2014 Microelectron. Eng. 11989

12. Wang S B, Xiong B F, Zhou S B, Huang G, Chen S H and Yi X J 2005 Sensors Actuat. A: Phys. 117110

13. Bilenberg B, Jacobsen S, Pastore C, Nielsen T, Enghoff S R, Jeppesen C, Larsen A V and Kristensen A 2005 Japn. J. Appl. Phys. 445606

14. Ziman J 1967 Sci. Am. 217180

15. Timmer B, Sparreboom W, Olthuis W, Bergveld P and van denBerg A 2002 R. Soc. Chem. 2121

16. Hus S, Van Den Broeke D J, Shi X, Fung Chen F, Knose W T, Corcoran N P, Vedula S, MacNaughton C W and Richie M 2002 Proceedings of SPIE, Photomask and Next-Generation Lithography Mask Technology IX $\mathbf{4 7 5 4} 373$

17. Seltmann R, Doleschal W, Gehner A, Kück H, Melcher R, Paufler J and Zimmer G 1996 Microelectron. Eng. 30123 